\title{
The Principles of Forming of the Mathematical Model of Nanoelectronic Components of Quantum Computer Systems with Memresistance Branches
}

\author{
Andrei Bondarev \\ department of industrial power supply \\ Kumertau branch of Orenburg State University \\ Kumertau, Russia \\ bondarevav@kfosu.edu.ru
}

\author{
Vladimir Efanov \\ department of electronics and biomedical technologies \\ Ufa State Aviation Technical University \\ Ufa, Russia \\ efanov@mail.ru
}

\begin{abstract}
The element base, based on the use of a variety of low-dimensional structures, is the most promising for new generations of electronic technology. However, in the transition to nanometer-scale systems, the quantum-mechanical nature of quasiparticles in a solid begins to manifest itself clearly. As a result, there is a situation when quantum effects begin to play a key role in physical processes in such objects and in the functioning of devices based on them. Since nanoelectronic components are components of modules and units of quantum computing systems, the task arises to study the physical processes and features of circuit design of electronic devices built on the basis of nanoelectronic structures. To this end, it is necessary to develop a generalized structuralparametric model of hybrid radioelectronic systems based on micro-and nanoelectronic components, taking into account the influence of uncertain factors of the external and internal environment, to evaluate the behavior of such complex devices in different operating conditions. In article features of forming of mathematical models of components of quantum computer systems with introduction to structure of the electric multiterminal network of the branches containing a new element of a nanoelectronics - the memristor are considered.
\end{abstract}

Keywords-Mathematical model, nanoelectronic components, memristor, quantum computer, multipole, matrix, set of elements.

\section{INTRODUCTION}

Development of information devices based on nanoelectronic structures belongs to the most relevant and dynamically developing field of modern science. Interest in this area is connected both with use of essentially new physical phenomena, and with the prospects of creation of new quantum devices and systems with broad functionality on the basis of already open physical regularities. Use of devices in which functioning quantum effects play a key role allows to increase sharply the capacity of computing systems, to increase the handling capacity of communication channels, to provide growth of data capacity and quality of systems of display of information with simultaneous decrease in energy consumption, it is essential to increase sensitivity of touch devices and to expand a range of the measured sizes and also to increase the specific weight of use of electronic and optoelectronic components in medical, biological, chemical, machinebuilding and other technologies.

The element base based on use of various lowdimensional structures is the most perspective for the electronic equipment of new generations. However, upon transition to the systems of nanometer scale the quantum mechanical nature of quasiparticles in a solid body begins to be shown distinctly. The situation when quantum effects begin to play a key role in physical processes in such objects and in functioning of devices on their basis results. The quantum computer can serve as one of the most known examples of quantum mechanical systems.

The quantum computer uses the phenomena of quantum superposition and quantum complexity. These are the cars based on the unique behavior described by quantum mechanics, and different from behavior of classical systems. One of such differences consists in use of quantum bits or qubits. Mathematically the qubit is a two-dimensional complex space that allows being to the quantum computer in a condition of quantum superposition. Already available 128-qubit quantum supercomputer of the Canadian company D-Wave shows exaFLOPS performance. However, quantum effects on which the D-Wave supercomputer is focused work at a temperature, near absolute to zero, about two tens milligradus Kelvin. In this regard, the problem of development of quantum devices, which do not demand similar service conditions, is relevant. Nanoelectronic components based on oxides in which the memristivny effect is observed are among such devices. The behavior of the memristor allows using it as a bipolyary key, an element of memory or a component of a logical element. When giving voltage of a certain polarity the memristor closes or disconnects the chain passing through it, i.e. the memristor passes from a state " 0 " into a state " 1 " and vice versa. Moreover, the memristor "remembers" this state and can store almost beyond all bounds long and for this purpose, it does not need voltage source. Time of switching of the memristor reached today from one fortune in another makes about $120 \mathrm{nc}$. 
At the same time there is a number of problems when using nanoelectronic structures in radio-electronic systems. These problems are generally connected both with designing of such structures, and with ensuring operability of electronic devices on their basis. Electronic systems have to work in the conditions of low or high temperatures, at the increased radiation level or influence of electric and magnetic fields, etc.

Extremely high cost of similar systems is caused, in addition to other causes as well the high cost of development and production of nanoelectronic components.

As nanoelectronic components are components of modules and knots of quantum computing systems, there is a research problem of physical processes and features of circuitry design of the electronic devices constructed based on nanoelectronic structures. For this purpose, it is necessary to develop the generalized structural and parametrical model of hybrid radio-electronic systems based on micro and nanoelectronic components taking into account influence of uncertain factors of external and internal environment to estimate behavior of similar complex devices in various service conditions.

In this direction the certain practical results, which are based on results of pilot studies, of course, are achieved. For the majority of the known nanoelectronic components the volt-ampere and other characteristics allowing to predict their behavior in these or those conditions are received, their mathematical models in the main operating modes are defined.

However, there are practically no researches directed to analytical process description of circuitry design of similar systems that would allow choosing the most suitable circuit option to estimate the level of compatibility of its components. All this will allow reducing, eventually, costs for prototyping at design of complex quantum computing systems.

In this regard, in this work an attempt to create a mathematical model of the electric multiterminal network for components of quantum electronics is made.

\section{FORMATIONS OF BASIC SET OF ELEMENTS OF NANOELECTRONIC STRUCTURES}

The mathematical model of the electric multiterminal network is formed based on a certain basic set of elements. The universal set of such elements includes: the linear and nonlinear resistor, linear and nonlinear capacity, linear and nonlinear inductance, independent sources of current and voltage, the dependent (operated) sources - the current source operated by current or voltage and voltage source operated by voltage or current. Thus, this set contains 12 elements which parametrical equations are known [1-12, 17-19].

However, here it is necessary to consider the assumption made by the American researcher Chua of L.O. in 1971 that there is the fourth Basic Element of electrical circuits - along with inductance, the capacity and the resistor. Chua recognized that there have to be ratios connecting all four main variables of electrical circuits: current of I, U voltage, a charge of $Q$ and a magnetic flux of F. Vsego of such ratios can be six. Five of them are well known. The charge is an integral on time from current. The law of electromagnetic induction of Faraday defines communication between voltage and a magnetic flux. Voltage and current are connected according to the law of Ohm through $\mathrm{R}$ resistance, a charge and voltage - through the capacity of $\mathrm{C}$, and a magnetic flux and current - through inductance of $\mathrm{L}$. There is no sixth ratio connecting a magnetic flux and a charge. Chua assumed that these sizes are connected through the "absent" element - the memristor possessing "memresistance" of M:
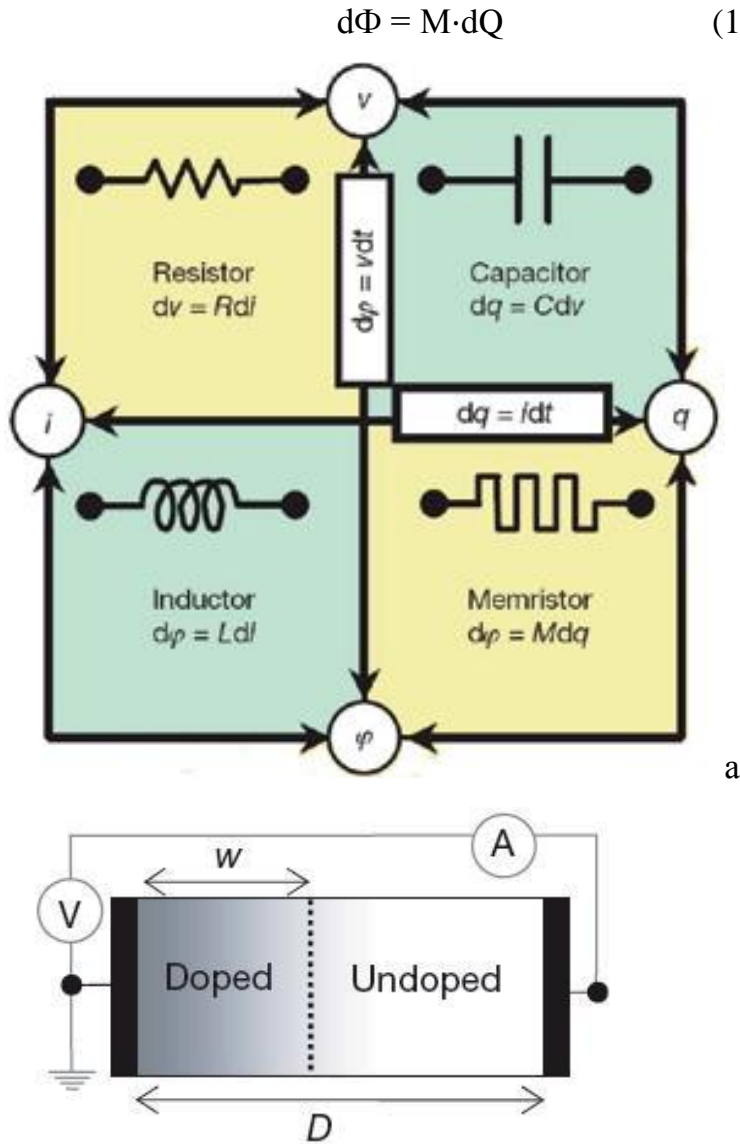

Undoped:

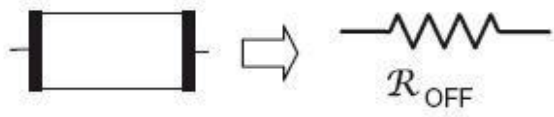

Doped:

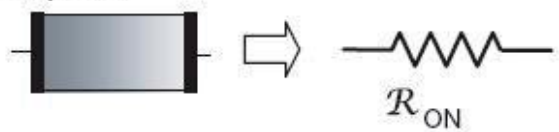

b) 


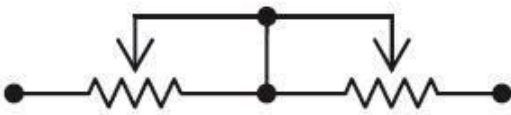

$\mathcal{R}_{\mathrm{ON}} W I D \quad \mathcal{R}_{\mathrm{OFF}} W I D$

Figure 1 - a) - basic elements of electrical circuits: resistor, condenser, inductance and memristor; b) - structure of the memristor; c) - equivalent scheme of the memristor [14]

We will note that in this case it is necessary to understand integral from voltage on time as a stream. Showed the Chu that generally the memresistance has to depend on a charge of $q$ [5.14].

The theoretical model of the memristor can be introduced as follows [14]:

$$
\mathrm{u}(\mathrm{t})=\left(R_{\text {ON }} \frac{w(\mathrm{t})}{D}+R_{\text {OFF }}\left(1-\frac{w(t)}{D}\right)\right) \cdot i(t)
$$

At the same time, the border is displaced under the law:

$$
\frac{d w(t)}{d t}=\mu_{W} \cdot \frac{R_{D N}}{D} \times i(t),
$$

where $\mu_{u}-$ average mobility of ions.

Integration gives a formula for $w(t)$ :

$$
w(t)=\mu_{u} \cdot \frac{R_{D N}}{D} \cdot q(t),
$$

Considering that $\mathrm{RON}<<\mathrm{ROFF}$, we receive expression for a memresistance [13]:

$$
\left.M(q)=R_{\text {OFF }}\left(1-\frac{\mu_{M} \cdot R_{D N}}{D^{2}}\right)\right) \cdot q(t) .
$$

More than a quarter of the century, the memristor remained the hypothetical element of a chain, which does not have material realization. However, in 2008 the group of researchers of the HP Company led by Stanley Williams at last created the real memristor. Its properties corresponded to the model offered Chua. The memristor developed by Williams's group was the thin layer of semiconductor material placed between two metal contacts. On the one hand a layer there is an alloying impurity (positive ions) - in the field of width ${ }^{w}[14$, $15]$.

\section{ANALYSIS OF CHARACTERISTICS OF THE MEMRISTOR}

Because the memristor from the category of hypothetical components passed into the field of implementation and the leading producers of nanoelectronics apply already considerable time, it makes sense to look at structure of mathematical models of electric multiterminal networks in a new way. As characteristics of nanoelectronic components substantially depend on intensity of magnetic field, the adequate mathematical description of similar systems requires introduction to structure of basic set of elements of the linear and nonlinear memristor, which is responsible for processes of interrelation of electric charge and magnetic field in structure of nanoelectronic devices.

It is obvious that it is necessary to talk not about one, and about two new elements of basic set. It follows from the known experimental dependences of VAC received by the western producers. They showed if the alternating sinusoidal voltage of a certain frequency is attached to the memristor, its volt-ampere characteristic takes the form reminding a Lissajous figure with the center in origin of coordinates. Therefore, the memristor, unlike the resistor, possesses a hysteresis. With increase in frequency of voltage, the hysteresis curve degenerates in a straight line. The specified circumstance makes related the memristor with resistance, only in the return analogy (at high frequencies of VAC of the memristor becomes linear). Here therefore it is about two new elements of basic set: linear and nonlinear memristors.

\section{TOPOLOGICAL ANALYSIS ELECTRICAL MULTITERMINAL NETWORK.}

Earlier the mathematical model of the electric multiterminal network in hybrid basis of the topological and parametrical equations of standard components of basic set was already received [12]. With introduction of the memristor to this set of elements there is a need of creation of the new mathematical model considering not only internal communications and processes on current and voltage, but also on magnetic components. In addition, there is a new class of branches of the topological flowgraph - memresistance branches.

The generalized mathematical model of the multiterminal network will consist of $n$ topological and $n$ of the parametrical equations. Really, the topological equations for former basic set [12]:

$$
\left\{\begin{array}{l}
D \cdot I=0 \\
B \cdot U=0
\end{array}\right.
$$

Where D - matrix of the main sections (k-1) equations; $\mathrm{B}-$ matrix of the main contours $(\mathrm{n}-\mathrm{k}+1)$ equations; $\mathrm{n}$ - number of branches and $\mathrm{k}$ - number of knots; ${ }^{I}, U$ - currents and voltage in branches of directed graph of the scheme.

Decomposition form for vectors of currents and voltage:

$$
\left\{\begin{array}{c}
U=\left[U_{L}^{X}, U_{H}^{X}, U_{R}^{X}, U_{M^{\prime}}^{X}, U_{C}^{P}, U_{H}^{P}, U_{R}^{P}, U_{M}^{P}\right] \\
I=\left[I_{L}^{X}, I_{H^{\prime}}^{X} I_{R}^{X}, I_{M^{x}}^{X} I_{C}^{P}, I_{H^{p}}^{P} I_{R}^{P}, I_{M}^{P}\right]
\end{array},\right.
$$

where $U_{L}^{X}, I_{L}^{X}$ - voltage and currents on inductive chords; $U_{C}^{p}, I_{C}^{p}$ - voltage and currents on capacitive edges; ${ }^{X}, U_{R}^{P}, I_{R}^{P}, I_{R}^{X}$ - voltage and currents on resistive edges and chords; $U_{H}^{X}, U_{H}^{p}, I_{H}^{P}, I_{H}^{X}$ - voltage and currents on nonlinear edges and chords; ${ }_{M}^{X}, U_{M}^{P}, I_{M}^{X}, I_{M}^{p}$ - voltage and currents on the memresistance edges and chords.

The system of the topological equations concerning currents will take a form: 


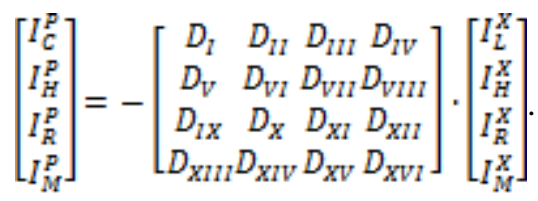

Concerning voltage:

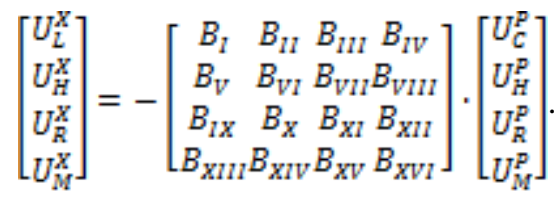

Subject to the foregoing, the set of the topological equations in hybrid basis of currents and voltage will consist of four groups of the equations:

$$
\begin{aligned}
& \int\left[\begin{array}{c}
U_{L}^{X} \\
I_{C}^{p}
\end{array}\right]=\left[\begin{array}{cc}
-B_{I} & 0 \\
0 & -D_{I}
\end{array}\right] \cdot\left[\begin{array}{c}
U_{C}^{p} \\
I_{L}^{X}
\end{array}\right]+\left[\begin{array}{cc}
-B_{I I} & 0 \\
0 & -D_{I I}
\end{array}\right] \cdot\left[\begin{array}{c}
U_{H}^{P} \\
I_{H}^{X}
\end{array}\right]+ \\
& +\left[\begin{array}{cc}
-B_{U I I} & 0 \\
0 & -D_{U I}
\end{array}\right] \cdot\left[\begin{array}{c}
U_{R}^{P} \\
I_{R}^{X}
\end{array}\right]+\left[\begin{array}{cc}
-B_{I V} & 0 \\
0 & -D_{I V}
\end{array}\right] \cdot\left[\begin{array}{c}
U_{M}^{p} \\
I_{M}^{X}
\end{array}\right] \\
& {\left[\begin{array}{c}
U_{f}^{X} \\
I_{H}^{P}
\end{array}\right]=\left[\begin{array}{cc}
-B_{V} & 0 \\
0 & -D_{V}
\end{array}\right] \cdot\left[\begin{array}{c}
U_{C}^{p} \\
I_{L}^{X}
\end{array}\right]+\left[\begin{array}{cc}
-B_{V I} & 0 \\
0 & -D_{V I}
\end{array}\right] \cdot\left[\begin{array}{c}
U_{H}^{p} \\
I_{H}^{X}
\end{array}\right]+} \\
& +\left[\begin{array}{cc}
-B_{V I I} & 0 \\
0 & -D_{V I I}
\end{array}\right] \cdot\left[\begin{array}{c}
U_{R}^{p} \\
I_{R}^{X}
\end{array}\right]+\left[\begin{array}{cc}
-B_{V I I I} & 0 \\
0 & -D_{V I I I}
\end{array}\right] \cdot\left[\begin{array}{l}
U_{M}^{P} \\
I_{M}^{X}
\end{array}\right] \\
& {\left[\begin{array}{c}
U_{R}^{X} \\
I_{R}^{p}
\end{array}\right]=\left[\begin{array}{cc}
-B_{I X} & 0 \\
0 & -D_{I X}
\end{array}\right] \cdot\left[\begin{array}{c}
U_{C}^{p} \\
I_{L}^{X}
\end{array}\right]+\left[\begin{array}{cc}
-B_{X} & 0 \\
0 & -D_{X}
\end{array}\right] \cdot\left[\begin{array}{c}
U_{R}^{P} \\
I_{R}^{X}
\end{array}\right]+} \\
& +\left[\begin{array}{cc}
-B_{X I} & 0 \\
0 & -D_{X I}
\end{array}\right] \cdot\left[\begin{array}{c}
U_{R}^{P} \\
I_{R}^{X}
\end{array}\right]+\left[\begin{array}{cc}
-B_{X I I} & 0 \\
0 & -D_{X I I}
\end{array}\right] \cdot\left[\begin{array}{c}
U_{M}^{P} \\
I_{M}^{X}
\end{array}\right] \\
& {\left[\begin{array}{c}
U_{M}^{X} \\
I_{M}^{p}
\end{array}\right]=\left[\begin{array}{cc}
-B_{X I I I} & 0 \\
0 & -D_{X I I}
\end{array}\right] \cdot\left[\begin{array}{c}
U_{C}^{p} \\
I_{L}^{X}
\end{array}\right]+\left[\begin{array}{cc}
-B_{X I V} & 0 \\
0 & -D_{X I V}
\end{array}\right] \cdot\left[\begin{array}{l}
U_{H}^{p} \\
I_{H}^{X}
\end{array}\right]+} \\
& +\left[\begin{array}{cc}
-B_{X V} & 0 \\
0 & -D_{X V}
\end{array}\right] \cdot\left[\begin{array}{c}
U_{R}^{P} \\
I_{R}^{X}
\end{array}\right]++\left[\begin{array}{cc}
-B_{X V I} & 0 \\
0 & -D_{X V I}
\end{array}\right] \cdot\left[\begin{array}{l}
U_{M}^{p} \\
I_{M}^{X}
\end{array}\right]
\end{aligned}
$$

\section{PARAMETRICAL DESCRIPTION OF THE MEMRISTOR}

We will pass to consideration of the parametrical equations now.

Expressions

for $U_{L}^{X}, U_{H}^{X}, U_{R}^{X}, U_{C}^{p}, U_{H}^{p}, U_{R}^{p}$ и $I_{L}^{X}, I_{H}^{X}, I_{R}^{X}, I_{C}^{p}, I_{H}^{p}, I_{R}^{p}$ are received earlier and known [12]. Expressions are unknown for $U_{M}^{X}, U_{M}^{p}, I_{M}^{X}, I_{M}^{p}$.
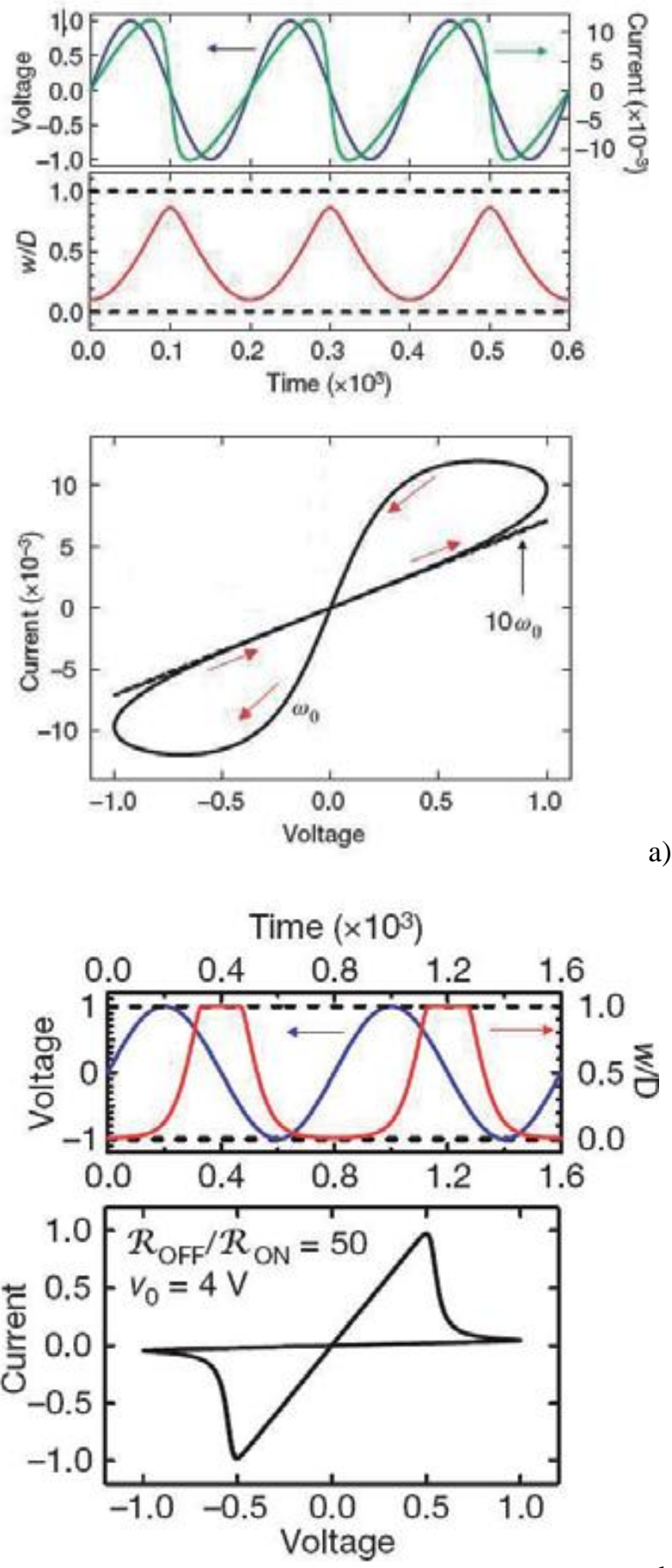

b)

Figure 2 - Results of behavior modeling of the memristor: a) - on the basis of the model described by the equations (2-5); b) - at multiplication of the right member of equation (3) by window function w(1-w)/D. On axes dimensionless values of voltage, current and time, rated are postponed on $\mathrm{v} 0=1 \mathrm{~V}$ (fig.2,a) and according to $4 \mathrm{~V}$ (fig.2,b), i0 $=10 \mathrm{~mA}, \mathrm{t} 0=10 \mathrm{~ms}$ [14].

We will consider the parametrical equation of the linear memristor. This equation has the appearance similar to the law of Ohm [18]:

$$
u(t)=M(q) \cdot i(t)
$$

Further we will consider the nonlinear memristor having VAC similar to a Lissajous figure (see figure 2, 
a). In the nominal mode, the nonlinear memristor will be described by the following system of equations:

$$
\left\{\begin{array}{c}
u(t)=\alpha \cdot \sin \omega t \\
i(t)=\beta \cdot \sin (\omega t+\varphi)^{x}
\end{array}\right.
$$

where ${ }^{\alpha}$ and $\beta$ - permanent coefficients of width of a loop of a figure VAC; ${ }^{\omega}$ - frequency of current and voltage; $\varphi$ - figure slope angle VAC.

Having used standard decomposition of a sine of the amount, substituting the first equation of a system (12) in the second and expressing a function cosine through a sine, we will receive expression for the nonlinear memristor in the nominal mode:

$$
i(t)=u(t) \cdot \frac{\beta}{\alpha} \cdot \cos \varphi-u(t) \cdot \frac{\beta}{\alpha} \cdot \sin \varphi \cdot \sqrt{1-\frac{u^{2}(t)}{\alpha^{2}}} \cdot(1
$$

Taking into account the received ratios the system of the hybrid equations for linear the memresistance of branches will take the following form:

$$
\left[\begin{array}{c}
U_{M}^{X} \\
I_{M}^{P}
\end{array}\right]=\left[\begin{array}{cc}
0 & \hat{M} \\
\hat{M}^{-1} & 0
\end{array}\right] \cdot\left[\begin{array}{c}
U_{M}^{P} \\
I_{M}^{X}
\end{array}\right],
$$

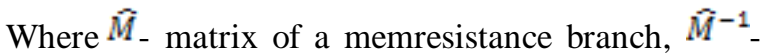
matrix of the return memresistance of a branch.

The system of the hybrid equations for nonlinear the memresistance of branches:

$$
\left[\begin{array}{c}
U_{M H}^{X} \\
I_{M H}^{P}
\end{array}\right]=\left[\begin{array}{cc}
0 & \hat{M} \\
\hat{M}^{-1} & 0
\end{array}\right] \cdot\left[\begin{array}{c}
U_{M H}^{P} \\
I_{M H}^{X}
\end{array}\right],
$$

\section{FORMING OF MODEL OF THE ELECTRIC MULTITERMINAL NETWORK}

For creation of a mathematical model of the electric multiterminal network in hybrid basis of the topological and parametrical equations we will use earlier received expressions [12].

Introduction to structure of basic set of elements of linear and nonlinear memristors will not affect properties of a reactivity of resistive branches of the multiterminal network. Therefore, expressions for them will remain invariable.

For jet branches:

$$
\left[\begin{array}{c}
U_{L}^{X} \\
I_{C}^{P}
\end{array}\right]=\left[\begin{array}{ll}
0 & \hat{L} \\
\hat{C} & 0
\end{array}\right] \cdot \frac{d}{d t}\left[\begin{array}{c}
U_{C}^{P} \\
I_{L}^{X}
\end{array}\right],
$$

Where $\hat{L}=\left[\begin{array}{cc}L_{k} & 0 \\ 0 & L_{l}\end{array}\right]$ - matrix of inductance $\left(k=\overline{1_{s} n_{\pi L}^{X}}\right.$, $\left.l=\overline{1_{s} n_{H L}^{X}}, n_{i l L}^{X}+n_{H L}^{X}=\operatorname{dim} U_{L}^{X}\right) ; \hat{C}=\left[\begin{array}{cc}C_{k} & 0 \\ 0 & C_{\mathfrak{l}}\end{array}\right]$ - matrix of capacities $(k$

$$
\left.=\overline{1_{s} n_{\pi C}^{X}}, l=\overline{1_{s} n_{H C}^{X}}, n_{\pi C}^{X}+n_{H C}^{X}=\operatorname{dim} I_{C}^{p}\right) \text { [12]. }
$$

For resistive branches:

$$
\left[\begin{array}{c}
U_{R}^{X} \\
I_{R}^{p}
\end{array}\right]=\left[\begin{array}{cc}
0 & \hat{R} \\
\hat{Y} & 0
\end{array}\right] \cdot\left[\begin{array}{c}
U_{R}^{P} \\
I_{R}^{X}
\end{array}\right]
$$

Where $\hat{R}$ - matrix of resistance of a branch, $\hat{Y}$ matrix of a conductive branch [12].

In addition, for nonlinear branches taking into account nonlinear memresistances (15) the system of the hybrid equations will take a form:

$$
\left[\begin{array}{c}
U_{H}^{X} \\
I_{H}^{p}
\end{array}\right]=\left[\begin{array}{cc}
M^{-1} & Z_{X}\left(I_{H}^{X}\right) \\
G_{P}\left(U_{H}^{p}\right) & M
\end{array}\right] \cdot\left[\begin{array}{c}
U_{H}^{P} \\
I_{H}^{X}
\end{array}\right],
$$

Where $Z_{X}\left(I_{H}^{X}\right){ }_{и} G_{P}\left(U_{H}^{P}\right)$ - scalar matrixes of equivalent resistances and conductances; $M^{-1}$ and $M_{\text {- }}$ scalar matrixes of equivalent return and direct memresistances.

As the left members of equation (18) and the second equation of a system (10) are equal, having equated their right parts, we will receive:

$$
\left[\begin{array}{c}
U_{H}^{P} \\
I_{H}^{X}
\end{array}\right]=A_{1}\left[\begin{array}{c}
U_{C}^{P} \\
I_{L}^{X}
\end{array}\right]+A_{2}\left[\begin{array}{c}
U_{R}^{p} \\
I_{R}^{X}
\end{array}\right]+A_{a}\left[\begin{array}{c}
U_{M}^{P} \\
I_{M}^{X}
\end{array}\right]
$$

where

$A_{1}=\left[\begin{array}{cc}M^{-1} & Z_{X}\left(I_{H}^{X}\right) \\ G_{p}\left(U_{H}^{P}\right) & M\end{array}\right] \cdot\left[\begin{array}{cc}-B_{V} & 0 \\ 0 & -D_{V}\end{array}\right]$,

$A_{2}=\left[\begin{array}{cc}M^{-1} & Z_{X}\left(I_{H}^{X}\right) \\ G_{P}\left(U_{H}^{p}\right) & M\end{array}\right] \cdot\left[\begin{array}{cc}-B_{V I I} & 0 \\ 0 & -D_{V I I}\end{array}\right]$,

$A_{a}=\left[\begin{array}{cc}-B_{\text {VIII }} & 0 \\ 0 & -D_{\text {VIII }}\end{array}\right]$

Because the right parts of the third equation of a system (10) and the equation (17) are equal, bringing similar members we will receive the following expression:

$$
\left[\begin{array}{c}
U_{R}^{P} \\
I_{R}^{X}
\end{array}\right]=G_{1}\left[\begin{array}{c}
U_{C}^{P} \\
I_{L}^{X}
\end{array}\right]+G_{2}\left[\begin{array}{c}
U_{H}^{P} \\
I_{H}^{X}
\end{array}\right]+G_{a}\left[\begin{array}{c}
U_{M}^{P} \\
I_{M}^{X}
\end{array}\right],
$$

where

$$
\begin{aligned}
G_{1} & =\left[\begin{array}{cc}
B_{X I} & \hat{R} \\
\hat{Y} & D_{X I}
\end{array}\right]^{-1} \cdot\left[\begin{array}{cc}
-B_{I X} & 0 \\
0 & -D_{I X}
\end{array}\right], \\
G_{2} & =\left[\begin{array}{cc}
B_{X I} & \hat{R} \\
\hat{Y} & D_{X I}
\end{array}\right]^{-1} \cdot\left[\begin{array}{cc}
-B_{X} & 0 \\
0 & -D_{X}
\end{array}\right], \\
G_{2} & =\left[\begin{array}{cc}
B_{X I} & \hat{R} \\
\hat{Y} & D_{X I}
\end{array}\right]^{-1} \cdot\left[\begin{array}{cc}
-B_{X I I} & 0 \\
0 & -D_{X I I}
\end{array}\right] .
\end{aligned}
$$

We will compare the fourth equation of a system (10) and expression (14) and, applying similar reasonings, as to resistive branches, we will receive:

$$
\left[\begin{array}{c}
U_{M}^{P} \\
I_{M}^{X}
\end{array}\right]=S_{I}\left[\begin{array}{c}
U_{C}^{P} \\
I_{L}^{X}
\end{array}\right]+S_{2}\left[\begin{array}{c}
U_{H}^{P} \\
I_{H}^{X}
\end{array}\right]+S_{a}\left[\begin{array}{c}
U_{R}^{P} \\
I_{R}^{X}
\end{array}\right],
$$

where

$$
\begin{aligned}
& S_{I}=\left[\begin{array}{cc}
-B_{X V I} & \widehat{M} \\
\hat{M}^{-1} & -D_{X V I}
\end{array}\right]^{-1} \cdot\left[\begin{array}{cc}
-B_{X I I I} & 0 \\
0 & -D_{X I I I}
\end{array}\right], \\
& S_{2}=\left[\begin{array}{cc}
-B_{X V I} & \widehat{M} \\
\hat{M}^{-1} & -D_{X V I}
\end{array}\right]^{-1} \cdot\left[\begin{array}{cc}
-B_{X I V} & 0 \\
0 & -D_{X I V}
\end{array}\right], \\
& S_{a}=\left[\begin{array}{cc}
-B_{X V I} & \widehat{M} \\
\hat{M}^{-1} & -D_{X V I}
\end{array}\right]^{-1} \cdot\left[\begin{array}{cc}
-B_{X V} & 0 \\
0 & -D_{X V}
\end{array}\right] .
\end{aligned}
$$


Comparing the right parts of the first of an equation of system (10) and (16), we will receive expression in hybrid basis for jet branches:

$$
\left[\begin{array}{ll}
0 & \hat{L} \\
\hat{C} & 0
\end{array}\right] \cdot \frac{d}{d t}\left[\begin{array}{c}
U_{C}^{P} \\
I_{L}^{X}
\end{array}\right]=Q_{1}\left[\begin{array}{c}
U_{C}^{P} \\
I_{L}^{X}
\end{array}\right]+Q_{2}\left[\begin{array}{c}
U_{H}^{P} \\
I_{H}^{X}
\end{array}\right]+Q_{a}\left[\begin{array}{c}
U_{M}^{P} \\
I_{M}^{X}
\end{array}\right],
$$

where

$$
\begin{aligned}
& Q_{1}=\left[\begin{array}{cc}
-B_{I} & Z_{3 \mathrm{~KB}} \\
V_{3 \mathrm{~KB}} & -D_{I}
\end{array}\right]+\left[\begin{array}{cc}
-B_{I V} & 0 \\
0 & -D_{I V}
\end{array}\right] \cdot G_{1}, \\
& Q_{2}=\left[\begin{array}{cc}
-B_{U I} & 0 \\
0 & -D_{I I}
\end{array}\right]+\left[\begin{array}{cc}
-B_{I V} & 0 \\
0 & -D_{I V}
\end{array}\right] \cdot G_{2} \\
& Q_{a}=\left[\begin{array}{cc}
-B_{U I U} & 0 \\
0 & -D_{U I U}
\end{array}\right]+\left[\begin{array}{cc}
-B_{U V} & 0 \\
0 & -D_{I V}
\end{array}\right] .
\end{aligned}
$$

Then we will compare the second equation of a system (10) and expression (18), we will receive:

$$
P_{1}\left(I_{H}^{X}, U_{H}^{P}\right) \cdot\left[\begin{array}{c}
U_{H}^{P} \\
I_{H}^{X}
\end{array}\right]=P_{2} \cdot\left[\begin{array}{c}
U_{C}^{P} \\
I_{L}^{X}
\end{array}\right]
$$

where

$$
\begin{aligned}
& P_{1}\left(I_{H}^{X}, U_{H}^{P}\right)=\left[\begin{array}{cc}
B_{V} & Z_{X}\left(I_{H}^{X}\right) \\
G_{p}\left(U_{H}^{P}\right) & D_{V}
\end{array}\right]+\left[\begin{array}{cc}
-B_{V I} & 0 \\
0 & -D_{V I}
\end{array}\right] \cdot G_{2}, \\
& P_{2}=\left[\begin{array}{cc}
-B_{I V} & 0 \\
0 & -D_{I V}
\end{array}\right]+\left[\begin{array}{cc}
-B_{V I} & 0 \\
0 & -D_{V I}
\end{array}\right] \cdot G_{1} .
\end{aligned}
$$

Uniting expressions (19-23), we will receive a fullsize mathematical model of the electric multiterminal network with memresistance branches in complete hybrid basis with use of a decomposition method of branches which will look as follows:

$$
\left\{\begin{array}{c}
{\left[\begin{array}{cc}
0 & \hat{L} \\
\hat{C} & 0
\end{array}\right] \cdot \frac{d}{d t}\left[\begin{array}{c}
U_{C}^{P} \\
I_{L}^{X}
\end{array}\right]=Q_{1}\left[\begin{array}{l}
U_{C}^{P} \\
I_{L}^{X}
\end{array}\right]+Q_{2}\left[\begin{array}{c}
U_{H}^{P} \\
I_{H}^{X}
\end{array}\right]+Q_{a}\left[\begin{array}{c}
U_{M}^{P} \\
I_{M}^{X}
\end{array}\right]} \\
{\left[\begin{array}{c}
U_{H}^{P} \\
I_{H}^{X}
\end{array}\right]=A_{1}\left[\begin{array}{c}
U_{C}^{P} \\
I_{L}^{X}
\end{array}\right]+A_{2}\left[\begin{array}{c}
U_{R}^{P} \\
I_{R}^{X}
\end{array}\right]+A_{a}\left[\begin{array}{c}
U_{M}^{P} \\
I_{M}^{X}
\end{array}\right]} \\
{\left[\begin{array}{c}
U_{R}^{P} \\
I_{R}^{X}
\end{array}\right]=G_{1}\left[\begin{array}{c}
U_{C}^{P} \\
I_{L}^{X}
\end{array}\right]+G_{2}\left[\begin{array}{c}
U_{H}^{P} \\
I_{H}^{X}
\end{array}\right]+G_{a}\left[\begin{array}{c}
U_{M}^{P} \\
I_{M}^{X}
\end{array}\right]} \\
{\left[\begin{array}{c}
U_{M}^{P} \\
I_{M}^{X}
\end{array}\right]=S_{I}\left[\begin{array}{l}
U_{C}^{P} \\
I_{L}^{X}
\end{array}\right]+S_{2}\left[\begin{array}{c}
U_{H}^{P} \\
I_{H}^{X}
\end{array}\right]+S_{a}\left[\begin{array}{c}
U_{R}^{P} \\
I_{R}^{X}
\end{array}\right]}
\end{array}\right.
$$

\section{CONCLUSION}

Expression (24) can be used at circuitry design and the analysis of schemes of nanoelectronic devices, and element base of quantum computers. Methods of the solution of the received mathematical model are subject to a further research with use of the device of interval arithmetics.

\section{REFERENCES}

[1] Bondarev A.V. Research problem of a robustness of electronic schemes by methods of interval calculations in the conditions of uncertainty/ Всборнике: CSIT'2015 Proceedings of the 17th International Workshop on Computer Science and Information Technologies. 2015. p. 145-149.

[2] Bondarev A.V., Muravyova E.A., Kadyrov R.R., Rahman P.A. The analysis of opportunities of construction and use of avionic systems based on COTS-modules/ARPN Journal of Engineering and Applied Sciences. 2016. V. 11. № 1. p. 78-92.

[3] Borghetti J., Snider G.S., Kuekes P.J. et al. 'Memresistance' switches enable 'stateful' logic operations via material implication. Nature letters, 2010, v.464, p.873-876

[4] Bourzac K. Memristor Memory Readied for Production. www.technologyreview.com/computing/25018/.

[5] Chua L.O. Memristor - the missing circuit element. - IEEE Trans. CircuitTheory, 1971, v.18, p.507-519.

[6] Johnson R.C. End of the CPU? HP demos configurable memristor. 4/9/2010, www.eetimes.com/electronicsnews/4088557/End-of-theCPU-HP-demos-configurablememristor.

[7] Kuekes P. J., Snider G. S., Williams R. S. Crossbar nanocomputers - Scientific American, 2005, v.293, p.72-78.

[8] Markoff J. H.P. Sees a Revolution in Memory Chip. www.nytimes.com/2010/04/08/science/08chips.html?_r=1.

[9] Memristor. - en.wikipedia.org/wiki/Memristor.

[10] Merritt R. HP researcher predicts memory-centric processors. 6/2/2010, www.eetimes.com/electronicsnews/4199856/HPresearcher-predicts-memory-centricprocessors

[11] Strukov D.B., Snider G.S., Stewart D.R., Williams R. S. The missing memristor found. - Nature letters, 2008, v.453, p.80-83.

[12] Bondarev A.V. The system of decision support at assessment of robustness of complex onboard radio-electronic systems based on COTS modules/ dissertation for a degree of the candidate of technical science / the Ufa state aerotechnical university. Ufa, 2011.

[13] Valiyev K. A. Quantum computers and quantum calculations//UFN. $-2012$.

[14] Valiyev K. A., Kokin A. A. Quantum computers: hopes and reality. The $\mathrm{m}$ is Izhevsk: Regular and chaotic dynamics, 2013. - 320 p.

[15] Yeliseyev N. Memristors and krossbara: nanotechnologies for processors / ELECTRONIC ENGINEER: Science, Technology, Business. № 8, 2010. - p.: 84-89.

[16] Zebrev G.I. Physical bases of a silicon nanoelectronics: Education guidance. - M.: MIFI, 2008. - $288 \mathrm{p}$

[17] Sigorsky V. P., A.I. Petrenko. Analysis algorithms of electronic schemes. - M.: Soviet radio, 1976, 608 p.

[18] Chua L. O., Lin Pen-min. Machine analysis of electronic schemes. Algorithms and computing methods. The lane with English - M.: Energy, 1980, $640 \mathrm{p}$

[19] Shuka A.A. Nanoelektronik's pike [electronic resource]: the education guidance under the editorship of A.S. Sigov. - the 2nd prod. (эл.). - M.: BINOMIAL. Laboratory of knowledge, 2012. 342 pages. - (Nanotechnologies). 